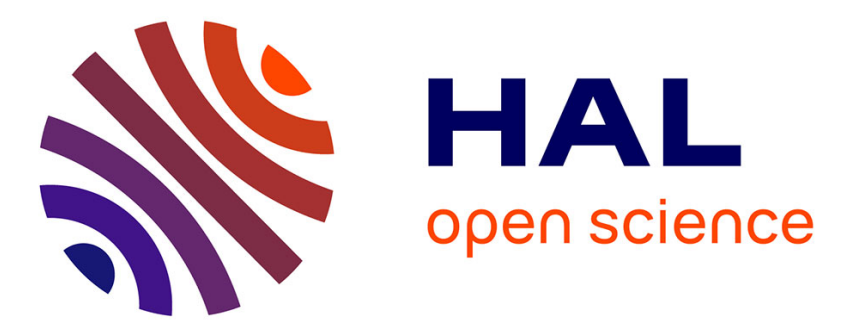

\title{
CSR ecological strategies and plant mating systems: outcrossing increases with competitiveness but stress-tolerance is related to mixed mating
}

François Munoz, Cyrille Violle, Pierre-Olivier Cheptou

\section{- To cite this version:}

François Munoz, Cyrille Violle, Pierre-Olivier Cheptou. CSR ecological strategies and plant mating systems: outcrossing increases with competitiveness but stress-tolerance is related to mixed mating. Oikos, 2016, 125 (9), pp.1296-1303. 10.1111/oik.02328 . hal-01354995

\author{
HAL Id: hal-01354995 \\ https://hal.science/hal-01354995
}

Submitted on 22 Aug 2016

HAL is a multi-disciplinary open access archive for the deposit and dissemination of scientific research documents, whether they are published or not. The documents may come from teaching and research institutions in France or abroad, or from public or private research centers.
L'archive ouverte pluridisciplinaire HAL, est destinée au dépôt et à la diffusion de documents scientifiques de niveau recherche, publiés ou non, émanant des établissements d'enseignement et de recherche français ou étrangers, des laboratoires publics ou privés. 
CSR ecological strategies and plant mating systems: outcrossing increases with competitiveness but stress-tolerance is related to mixed mating

François Munoz $^{1,2}$, Cyrille Violle ${ }^{1}$ and Pierre-Olivier Cheptou ${ }^{1}$

${ }^{1}$ CEFE UMR 5175, CNRS, Univ. de Montpellier, Univ. Paul-Valéry, Montpellier, EPHE, 1919 Route de Mende, FR-34293 Montpellier Cedex 5, France

${ }^{2}$ Univ. Montpellier 2, UMR AMAP, Boulevard de la Lironde, TA A51/PS2, FR-34398 Montpellier Cedex

5, France

Corresponding author: F. Munoz, Univ. Montpellier 2, UMR AMAP, Boulevard de la Lironde, TA A51/PS2, FR-34398 Montpellier Cedex 5, France. E-mail: francois.munoz@cirad.fr

Decision date: $30-\mathrm{Nov}-2015$

This article has been accepted for publication and undergone full peer review but has not been through the copyediting, typesetting, pagination and proofreading process, which may lead to differences between this version and the Version of Record. Please cite this article as doi: [10.1111/oik.02328]. 


\begin{abstract}
A number of plant traits influence the success of fertilization and reproduction in plants. Collectively these traits represent ecological syndromes that are of evolutionary significance. However, while an association between mating system and colonizing ability has been proposed, the existence of a broader relationship between mating system and a species' position in ecological succession has not been extensively investigated. Grime's CSR theory stresses that an ecological succession can involve changes from colonizing to either competitive or stress-tolerant strategies. How distinct dimensions of competitiveness and stress tolerance covary with mating systems has still not been considered. We designed a comparative approach to evaluate the link between mating system, life form and CSR strategies for 1996 herbaceous and woody species. We found that CSR strategies are significantly related to mating systems. Ruderal species - colonizers in early succession- were mostly selfers while more competitive species were more often outcrossers. On the other hand, greater physiological stress tolerance was associated with mixed mating systems. Outcrossing is classically expected to be advantageous for most life history strategies other than colonizers, but we suggest that reproductive assurance can counterbalance this effect in stressful environments where populations are sparse and pollinators are rare. Therefore, our results emphasize that competition and abiotic stresses are not equivalent selective pressures on the evolution of mating systems. Finally, we found plant life span to convey additional information on mating system variation, supporting its role for mating system evolution. These findings encourage further investigation of the evolutionary role of ecological strategies as syndromes of traits and suggest that the emergence of large databases of plant traits will help address the major evolutionary hypotheses on such syndromes.
\end{abstract}




\section{Introduction}

By governing gene transmission, mating system has major influence on individual fitness and the distribution of genetic variability. It thus determines the potential for adaptation and is central to our understanding of plant evolutionary processes. In addition, outcrossing rates have been shown to vary among populations and species in seed plants (Vogler and Kalisz 2001). Both ecological and genetic factors influence mating systems and several hypotheses have been put forward to explain this variation. The cost of outcrossing (3/2-transmission benefit of selfing genes, Fisher 1941), along with inbreeding depression, have been considered as primary drivers of mating system evolution by population geneticists (Lande and Schemske 1985, Lloyd 1979). Furthermore, pollen limitation due to pollination uncertainty, which is known to occur in natural populations (Knight et al. 2005), has been put forward as a major factor selecting for self-fertilization (Lloyd 1992).

A number of plant functional traits determine the ability of individuals and populations to establish and persist in a given environment (Violle et al. 2007). These traits constitute distinct ecological strategies reflecting the evolution and adaptation of plants across diverse environments (Grime 1977, Westoby et al. 2002). Syndromes of traits related to colonizing ability and persistence are especially critical for plant ecology (Grime 1977). In line with colonizing ability, Baker (1955) hypothesized that self-fertilization should be advantageous in colonizing populations where pollinators or partners for mating are scarce (this hypothesis was to become "Baker's Law"), and thereby selfing should be predominant for colonizing species. Baker's Law has become influential thanks to its potentially wide application to various situations such as early-successional species, weeds or invasive species (Davis 2005). Further theoretical models have confirmed that reproductive assurance during colonization may favour selfers (see however Cheptou and Massol 2009, Dornier et al. 2008, Pannell and Barrett 1998). Another line of argument proposed by Stebbins $(1957,1958)$ states that selffertilization should be positively associated with colonizing ability, because those mating systems allow the rapid fixation of successful genotypes. However, Grant (1967) predicted that 
colonizers should outcross more because they "are associated with diverse and in most cases flexible genetic systems" and "develop where a heterotic advantage may be fully utilized in conjunction with some means of effective dissemination" (p. 291).

The association between colonizing ability and self-fertilization is supported by the fact that self-compatible populations are over-represented in species distribution margins and recently colonized areas (Busch 2005, Van Kleunen and Johnson 2007). But conflicting data exist, e.g., Sutherland (2004) found that invasive species are actually less self-compatible than non-invasive species in the United States. In addition, although unable to self-fertilize, dioecious plants have revealed a paradoxical association with long-range dispersal (Bawa 1980, Renner and Ricklefs 1995), and they have been found to be overrepresented on isolated islands (Sakai et al. 1995).

These conflicting results, however, include a variety of biotic and abiotic contexts in which colonizers establish, and represent varying constraints on their dispersal abilities. Therefore, a dichotomy of colonizing and non-colonizing plants does not capture the diversity of ecological strategies that are selected according to biotic and abiotic constraints. In this regard, a broader perspective is needed on how multiple ecological properties are shaped by evolutionary forces together with mating systems. We here consider Grime's $(1974,1977,1979)$ CSR theory of ecological strategies, which provides an integrated scheme based on three primary strategies, namely, competitive ability (C), physiological tolerance to stress (S) and ruderality, i.e., adaptation to disturbance (R). The diversity of CSR strategies in plant communities (Grime 1977) is intimately linked to ecological successions where a temporal shift from colonizers to locally persisting and more conservative species is observed in an ecosystem affected by a disturbance. In this regard, we can distinguish successions as either favouring more competitive or more stress-tolerant strategies, depending on the biotic and abiotic contexts (Caccianiga et al. 2006). Here we hypothesize that CSR strategies are related to mating system variation in plants, because they characterize the diversity of ecological factors affecting mating system evolution (Uyenoyama et al. 1993). 
Plant life form related to life span (short vs. long lived) and growth form (herbaceous vs. woody) has also been considered as a key driver of mating system evolution. Stebbins (1950) discussed the possibility that shorter-lived species may be more associated with selfing than longer-lived species, and more recent studies have revealed that this pattern is robust (Barrett et al. 1996). Longer-lived species can be less constrained by pollen limitation, because they can reproduce several times, and can incur adult inbreeding depression (Morgan et al. 1997), which both favour outcrossing. More recently, Snell and Aarssen (2005) addressed the 'timelimitation' hypothesis, which suggests that pollination and reproductive period may be shorter in annuals because they occupy ephemeral habitats, thus selecting for selfing annuals. The role of life span and growth form in mating system evolution is at first glance complementary to the role of ecological properties such as competition, ruderality and stress-tolerance. In order to clarify the link between these apparently separate lines of arguments, we therefore aimed at assessing the respective links between life forms, CSR ecological strategies and plant mating systems.

We investigated the relationship between mating systems, life forms and Grime's CSR ecological strategies for 1,996 European plant species distributed over diverse and broad successional gradients. More and more extensive data have become available on CSR strategies, thanks to simpler and more efficient methods to measure them (Grime et al. 2007, Hodgson et al. 1999), and large databases now provide a huge amount of information on a variety of traits, including mating systems (e.g., BiolFlor, Kühn et al. 2004). We designed a Generalized Linear Mixed Model (GLMM) to analyse the relationship between mating systems and ecological traits while acknowledging the influence of phylogenetic relatedness (Garamszegi 2014, Hadfield and Nakagawa 2010).

\section{Material and Methods}

We selected 1,996 plant species for which the BiolFlor database (Kühn et al. 2004, http://www.ufz.de/biolflor/index.jsp) provided detailed information on physiological and 
ecological traits from many sources. For the phylogeny-based analyses, we used the dated Daphne phylogenetic tree provided by Durka and Muchalski (2012), which included these 1,996 species. Branch lengths were calibrated in million years. All the statistical analyses were performed in R (R Core Team 2014).

\section{Plant mating system}

We classified plant mating system following the BiolFlor categorization, namely, obligatory selfers, facultative selfers, mixed mating plants, facultative outcrossers and obligatory outcrossers. These classes provided a categorical ordinal variable representing a hierarchy of plant mating system from selfing to outcrossing, through mixed mating systems. If several classes were provided in BiolFlor for a single plant species, we attributed the median one. BiolFlor data on mating systems mainly refer to published quantitative analyses. In order to check their reliability, we compared the BiolFlor ordinal index with quantitative estimates from independent surveys relying on genetic markers (progeny array analysis, see Appendix 1). Good correlation between the ordinal BiolFlor index and independent progeny-array estimates of outcrossing rates (Kendall $\tau=0.72, p<0.001$ ) led us to use the BiolFlor ordinal scale as a good first approximation for our large dataset.

\section{Plant ecological strategies}

Grime $(1974,1977,1979)$ and Grime et al. (1997) proposed a scheme of three

fundamental ecological strategies, competitive ability (C), physiological tolerance to stress (S) and adaptation to disturbance $(\mathrm{R})$. These fundamental strategies are the vertices of the famous CSR triangle. Complying with the method of Klotz (1984), the BiolFlor database provides 7 categories of ecological strategies, including the three primary C (competitiveness), S (stress tolerance) and R (ruderality) strategies, along with 4 intermediate, secondary types (namely, CR, CS, SR and CSR). Assignation to one of the ecological strategy in BiolFlor was based on life form, leaf persistence, leaf anatomy, maximum growth height, flowering period, pollination 
and dispersal characteristics, and ecological indicator values (Ellenberg et al. 1991). Although based on three fundamental strategies, the CSR triangle is a 2-dimensional scheme. The fundamental strategies are expected to be found at the extremes of ecological gradients. Specifically, a switch from $\mathrm{R}$ to $\mathrm{C}$ strategies is expected along an ecological succession in nonstressful environment, where vital resources are not limited (Navas et al. 2010). On the other hand, a switch from $\mathrm{R}$ to $\mathrm{S}$ strategies is expected along an ecological succession in stressful and resource-limited environment (Caccianiga et al. 2006). Therefore we modelled the two dimensions of the CSR triangle by taking the CR edge as a reference dimension of competitiveness from $\mathrm{R}$ to $\mathrm{C}$, and the axis orthogonal to the $\mathrm{CR}$ edge and going to $\mathrm{S}$ as a second independent dimension of stress-tolerance, as already proposed in Grime et al. (1997, see Fig. 6). We then projected the 7 types in BiolFlor onto the CR edge of the CSR triangle to define the position of species over a competitiveness index, with three possible levels, CR1 for the R and SR types, CR2 for CR, CSR, S, and CR3 for C and SC (Fig. 1). Ruderal plants are generally first to colonize disturbed lands and, in this regard, a low value of the competitiveness index was related to colonizing ability in early ecological succession. We likewise projected the 7 types orthogonally to the CR edge to position species on a stress-tolerance index, with four possible levels, S1 for R, CR and C, S2 for CSR, S3 for SR and SC and S4 for S (Fig. 1). The combination of the two indexes covered all the 7 basic strategies. Table 2 shows the relative proportions of mating systems according to the competitiveness and stress-tolerance indexes. There was no correlation between competitiveness and stress-tolerance (Kendall rank correlation, $\tau=0.01, p$ $=0.53$ ), indicating that these indexes indeed represented independent dimensions of plant ecological strategies.

\section{Plant life form and lifespan}

Life-form categorisation into annuals, herbaceous perennials and woody perennials is widely used in evolutionary biology to test hypotheses on ecological strategies involved in plant mating evolution (Barrett et al. 1996, Morgan et al. 1997, Snell and Aarssen 2005, 
Stebbins 1950). We classified the 1,996 plant species of our study into annuals A, herbaceous perennials HP, and woody perennials WP. The classification was based on the Raunkiaer (1934) index and on lifespan information provided in the BiolFlor database, so that annuals $A$ included therophytes, herbaceous perennials HP included the remaining non phanerophytes, i.e, most geophytes, cryptophytes and chamephytes, and finally woody perennials WP included phanerophytes. Table 2 shows the proportion of mating systems for each life form. Over the 3 $x 7=21$ possible combinations of 3 life forms and 7 CSR strategies, 18 were found in our dataset, namely, A:C, A:CR, A:CS, A:CSR, A:R , A:S, A:SR, HP:C, HP:CR, HP:CS, HP:CSR, HP:R, HP:S, HP:SR, WP:C, WP:CS, WP:CSR and WP:S. Table 1a shows species numbers per life form and per level of competitiveness (CR levels of Fig.1) and Table $1 \mathrm{~b}$ the numbers per life form and per level of stress-tolerance (S levels of Fig.1).

A key issue was to address whether the variation in mating systems was related to the variation in ecological strategies as measured by the competitiveness and stress-tolerance indexes, in combination or not with life-form variation. Basically, life form was correlated to both competitiveness and stress-tolerance (Spearman rank tests, $p<0.001$; Table 1 ), and we could not assess any effect of life-form among ruderals (for CR1, 305 individuals are in A, but only 6 in $\mathrm{HP}$ and 0 in WP), or, conversely, any effect of the variation in competitiveness within woody perennials (224 individuals in CR3, but only 15 in 2 and 0 in 1). We could still analyze the variation of mating systems according to competitiveness for a given life form (e.g., among herbaceous perennials), or according to life form for a given level of competitiveness (e.g., for CR2).

\section{MCMC Generalized Linear Mixed Model}

Comparing plant traits and mating systems required addressing whether species display correlated features because of their phylogenetic relatedness (phylogenetic signal, see Blomberg and Garland 2002). We used the function fitDiscrete in R package geiger to fit a macroevolutionary model of the ordinal mating system variable, depending on the structure of 
the phylogeny. Pagel's $\lambda$ was used to represent the intensity of the phylogenetic signal in mating systems. The $\lambda$ parameter is a factor ranging between 0 and 1 , by which the lengths of internal branches in the phylogeny are multiplied. $\lambda=1$ then represents the structure of the original phylogeny, while $\lambda$ values closer to 0 represent longer terminal branches and ultimately a complete polytomy (star phylogeny). fitDiscrete performed an equal-rate, continuous-time Markov model of evolution and provided a maximum-likelihood for $\lambda=0.97$. We compared this model to a null model considering the absence of phylogenetic effect on mating system evolution, with $\lambda=0$. We performed a likelihood ratio test of the two models and found that the model with $\lambda=0.97$ performed significantly better than the model without phylogenetic structure $\left(\chi^{2}\right.$ test, $\left.p<0.001\right)$. Therefore a phylogenetic signal exists in the variation of mating systems and should reflect the imprint of evolutionary processes.

We then designed a Generalized Linear Mixed Model (GLMM) to address the influence of ecological strategies and life form on the ordinal categorization of mating systems, while taking into account the influence of phylogenetic structure. Mating systems were ordered with increasing outcrossing, and a cumulative link function was used to model the shifts between mating systems. The estimated cutpoints of the link function represented shifts to greater or lower outcrossing for varying ecological strategies and life forms. The model included a random term, for which the covariance was defined depending on the topology and branch lengths of the phylogeny (Hadfield and Nakagawa 2010). There is no close-form analytical solution for estimating parameters of such GLMMs, and we therefore performed a MonteCarlo Markov Chain (MCMC) simulation to calculate posterior probability distributions of the parameters. For this purpose, we used the MCMCgImm function of the R package MCMCgImmRAM (Hadfield 2014, Hadfield 2010, Hadfield 2015, Hadfield and Nakagawa 2010). The phylogenetic covariance structure was calculated using the function inverseA. The model included a single categorical predictor representing all the combinations of life form and CSR categories existing in our dataset, except the A:C, A:CS, HP:R, HP:SR and WP:S categories, which were too rare (i.e., less than 10 individuals). The analysis was thus restricted to 1,972 
species. We defined uninformative prior distribution of the fixed-effect parameters following a multivariate zero-mean Normal distribution with large variance. In the case of a categorical response, the variance of residuals should be fixed (here arbitrarily to 0.5 ) (Hadfield and Nakagawa 2010). We further used parameter expansion to improve MCMC performance (Gelman 2006, Hadfield 2014). The burn-in parameter of the MCMC simulation was adjusted to ensure that posterior distributions were estimated at the stationary state of the Markov Chain, that is, the first 10000 generations were withdrawn. The total number of MCMC iterations was set to 300000 , and the thinning parameter was set to 100 to control for autocorrelation in the Markov process. Therefore, 2900 sampled MCMC iterations were used to assess posterior distributions. We controlled convergence and autocorrelation of the resulting distributions using standard diagnostic tools of the coda package. We analyzed the predicted variation of mating systems with increasing competitiveness and stress-tolerance (Table $3 a$ and $3 b$ ), as well as according to life form (Table 3c). For instance, we calculated the combined response of all the levels of the predictor concerning R and SR strategies (CR1 level of competitiveness), and subtracted it to the response of CR, CSR and S strategies at CR2 level (see Fig. 1). The 95\% Highest Posterior Density (HPD) interval of this difference represented a change from CR1 to CR2 toward more outcrossing if positive, or toward more outcrossing if negative. If the interval included 0 , there was no predicted change. We likewise analyzed the variation of outcrossing depending on ecological strategies for a given life form, or depending on life form for a given ecological strategy. The R code used to perform MCMC modelling of the GLMM analysis is provided in Appendix 2a.

\section{Results}

The MCMC converged and displayed little autocorrelation with the selected parameters of simulations (burn-in, thinning and parameter expansion). The trace of MCMC estimations and the corresponding posterior distribution are provided for each category of the predictor in Appendix $2 \mathrm{~b}$. Phylogenetic structure contributed to the relationship between ecological 
predictors and mating system variation, as the $95 \%$ Highest Posterior Density (HPD) interval of the random phylogenetic effect did not include 0 (Appendix 2b, last plot). We further combined the estimated effects of each combination of life form and CSR strategy to assess the predicted variation of outcrossing along the competitiveness (Table 3a) and stress tolerance (Table 3b) indexes, and across life forms (Table 3c). We calculated the 95\% HPD intervals of the predicted differences between levels of the indexes (from a row category to a column category in Table 3). Positive intervals indicated a significant increase and negative intervals a significant decrease in outcrossing. We found increasing outcrossing along the competitiveness index (Table 3a). Conversely, we did not find consistent outcrossing variation along the stress-tolerance index. Highest stress-tolerance (S4 level in Fig.1) did not display more or less outcrossing that other categories of the index, and only weak variation was found from levels S1 to S2 (increase) and from S2 to S3 (decrease) (Table 3b). Therefore outcrossing did not increase with stress tolerance. Concerning life forms, we found increasing outcrossing from annuals (A) to woody perennials (WP), but woody perennials outcrossed only slightly more than herbaceous perennials (lower bound of the HPD interval close to 0) (Table 3c). An ecological succession in non-stressful abiotic environment would then display a clear variation from early-stage, more selfing, more annual and ruderal species to later-stage, more outcrossing, more perennial, more woody and competitive species. However, in physiologically more stressful environments, the absence of such an increase suggests that increasing physiological stress and competition do not have the same influence on mating systems. In addition, mixed mating was more frequent in stress-tolerant species, compared to competitive species (72\% of taxa at highest stress-tolerance level S4 were mixed mating, and $21 \%$ were outcrossing, while $58 \%$ of taxa at highest competitive level CR3 were mixed mating, while $41 \%$ were outcrossing, $\chi^{2} p<0.001$; Table 2).

Furthermore, we found that outcrossing increased with competitiveness within annuals (from CR1 to CR2, HPD interval = $[0.04 ; 0.82]$ ), and within herbaceous perennials (from CR2 to CR3, HPD interval $=[0.13 ; 0.74])$. We also found that outcrossing increased between annuals 
and herbaceous perennials for a fixed level of competitiveness (i.e., at CR2, HPD interval = $[1.78 ; 2.64])$, but not between most competitive herbaceous and woody perennials at CR3 (HPD interval $=[-0.14 ; 1.69])$. These nested variations were basically consistent with the general patterns of Table 3. It underlines that life form and CSR strategies are ecological properties related to plant mating system variation in a complementary way.

\section{Discussion}

Our results demonstrate how variation in plant ecological strategy and life form closely relates to variation in mating system. First, using a large dataset, we confirm that annuals are more selfing and perennials are more outcrossing (Tables 2 and 3), as previously advanced (Barrett et al. 1996). Second, we found that Grime's CSR strategies are related to mating system in two independent ways. On one hand, on the CR edge of the triangle representing a variation of competitiveness, ruderal selfers were replaced by more competitive outcrossers (Table 3a), as observed both at intraspecific and interspecific level along ecological successions (Cheptou et al. 2002, Rydin and Borgegard 1991, Silvertown et al. 2002). On the other hand, there was no increasing outcrossing along the orthogonal index of stress-tolerance (Table $3 b$ ), and mixed mating was frequent in most stress-tolerant species (Table 2). Third, we highlight that the CSR ecological strategies and the classical life form categorization based on life span variation complementarily influence the variation of plant mating systems.

Various hypotheses have been proposed for the association between mating system and ruderality. Our results are in line with Stebbins (1957)'s and Baker $(1955,1967)$ 's predictions that ruderal species are more selfing than competitive species. These authors however, proposed different selection pathways to explain the relationship. Stebbins hypothesized that the rapid fixation of locally adapted genotypes through apomixis or selfing should be a key selection factor, but this still needs further experimental support. Alternatively, focusing on the lack of pollinators, Baker's view conforms to the classical arguments for mating system evolution (Lloyd 1979). Many species are indeed pollen-limited in natural conditions (Burd 
1994) and ecological disturbance such as pollinator losses or plant density variation (Knight et al. 2005) are known to affect pollen limitation. In this context, selfing may provide reproductive assurance (Eckert et al. 2009, Kalisz et al. 2004). In our study however, inferences about species' successional status were derived from the CSR theory and do not include an analysis of dispersal or colonizing ability per se, since other traits linked to early succession position of species may play a role. Though appealing, the idea that colonizing species or species from early successional stages are more prone to pollen limitation than plants from later successional stages is, to our knowledge, not established and needs further experimental support. As a consequence, it is premature to consider our results as a support for Baker's law. In addition, previous studies focused on the relationship between mating system and dispersal ability, which is hypothesized to be higher in colonizers (see for instance Price and Jain 1981). However dispersal ability does not directly reflect colonizing ability, and other ecological properties determine establishment and survival of pioneer species. In this regard, the ruderality strategy of the CSR theory represents a more comprehensive index of colonizing ability. Our results then support the idea that the comprehensive nature of ecological strategies can capture the broad adaptive nature of plant mating system variation in plants.

It has been well established that inbreeding depression is a major factor selecting for outcrossing (Charlesworth et al. 1990, Lloyd 1979). High inbreeding depression is expected to select for outcrossing while low inbreeding depression is expected to select for selfing. Population geneticists have established that the level of inbreeding depression caused by deleterious mutations can be affected by selfing as a result of mutation/selection balance (purging, Charlesworth et al. 1990). How inbreeding depression influences the variation in mating systems along ecological successions has been mainly addressed at the intraspecific level. For example, Cheptou et al. (2000) found that the magnitude of inbreeding depression in populations of the ruderal annual species Crepis sancta, a partially self-incompatible plant species, increases along an ecological succession, and Cheptou et al. (2002) showed that it is associated with a slight increase in outcrossing rates and self-incompatibility. Cheptou et al. 
(2002) proposed that selfing may be selected at earlier stages, via automatic selection,

because of the 3/2-transmission benefit of selfing genes (Fisher 1941), while higher inbreeding depression in later stages counteracts the automatic advantage of selfing genes, thus favouring outcrossing. Interestingly, this does not exclude the role of reproductive assurance in early successional stages, but Cheptou et al. (2002) did not find any evidence of pollination limitation in early successional stages. In a compilation of surveys, Armbruster \& Reed (2005) highlighted that stress tends to increase the magnitude of inbreeding depression, but counterexamples for this trend were also reported. Their definition of environmental stress encompassed both the effect of competition and the effect of abiotic constraints, but these stresses could affect inbreeding depression in a different manner. The expression of inbreeding depression may therefore differ with respect to CSR strategies along the competitiveness and stress-tolerance indexes, in line with our distinct patterns of outcrossing variation. However, empirical data on whether and how inbreeding depression varies with CSR strategy is lacking. Therefore the contribution of inbreeding depression to broad-scale, interspecific variation in mating systems remains to be tested.

We further hypothesize that pollen limitation is an important constraint in a broader context when environmental conditions impose low population densities and short and uncertain reproductive periods. Specifically, most stress-tolerant species of the present study belonged to three main ecological contexts, high elevation/cold ecosystems, desert/salt places, and acid peat bogs. In these contexts, the reproductive period is constrained by the environment, pollinators are rare, populations are often sparse, and environmental stochasticity is high. Reproductive assurance may then select for selfing. Conversely, enhanced inbreeding depression in the context of high physiological stress could limit such directional selection and result in more frequent mixed mating system (Table 2).

We also found life form to contribute to mating system variation in addition to CSR ecological strategies. Outcrossing basically increases with life span, even though the variation between herbaceous and woody perennials is very small (Table 3c). We note here that various 
ecological strategies can exist for a given life form (Table 1) and relate to different mating systems, as found within annuals and herbaceous perennials. Conversely, different life forms can be found at a given level of competitiveness, and we found increasing outcrossing between annuals and herbaceous perennials at the CR2 level. The fact that life form contributed to the variation of outcrossing rates in addition to CSR strategies thereby provides support to hypotheses that state that inbreeding depression (Morgan et al. 1997) and reproductive assurance (Snell and Aarssen 2005) depend on life span. First, because of multiple episodes of selection against inbred individuals, inbreeding depression is expected to be higher in perennial species. A recent study has shown that almost one hundred percent of inbred progeny died before the adult stage in the mixed mating Rhododendron ferrugineum (Delmas et al. 2014). Second, pollination limitation should have less impact on perennials because of their possibility to postpone reproduction. Therefore, (i) different patterns of mating system variation with competitiveness and stress-tolerance show that the separate dimensions of CSR ecological strategies aid understanding mating system evolution, and (ii) the additional effect of annuality vs. perenniality supports the idea that time limitation is per se an important driver of mating system evolution.

The fact that the evolution of self-fertilization depends on genetic as well as ecological factors is well accepted (Barrett and Harder 1996). In this study, we have provided new pieces of evidence on how ecological traits pertaining to successional processes and stress tolerance relate to plant mating systems. These results encourage further analysis on how the leading dimensions of ecological trait variation (Westoby et al. 2002) influence the evolution of mating systems. On the other hand, mating system is still an overlooked plant trait in ecological studies. While plant traits related to resource acquisition, persistence and regeneration have been found to be good proxys of the mechanisms driving ecological successions (Huston and Smith 1987, Navas et al. 2010, Raevel et al. 2012), traits regarding mating systems have been neglected despite their broad variation along successions. We accordingly suggest adding mating systems to the list of traits of interest when analyzing the dynamics of ecological 
successions. Including more information on breeding systems and reproductive traits in global trait databases (Kattge et al. 2011) should stimulate future comparative studies and help bridge the gap between ecological and evolutionary theories.

\section{Acknowledgements}

We warmly thank the reviewers and the editor for their detailed comments and suggestions, which have greatly helped improve the manuscript. We also thank Xavier Morin, Antoine Dornier and Valérie Raevel for helpful discussion and suggestions. 


\section{References}

Armbruster, P. and Reed, D. H. 2005. Inbreeding depression in benign and stressful

environments. - Heredity 95: 235-242.

Baker, H. G. 1955. Self-compatibility and establishment after 'long-distance' dispersal. Evolution 11: 449-460.

Baker, H. G. 1967. Support for Baker's law-as a rule. - Evolution 21: 853-855.

Barrett, S. C. H. and Harder, L. D. 1996. Ecology and evolution of plant mating. - Trends Ecol. Evol. 11: 73-79.

Barrett, S. C. H., et al. 1996. The comparative biology of pollination and mating in flowering plants. - Phil. Trans. R. Soc. Lond. B 351: 1271-1280.

Bawa, K. S. 1980. Evolution of dioecy in flowering plants. - Annu. Rev. Ecol. Syst. 11: 15-39.

Blomberg, P. and Garland, T. 2002. Tempo and mode in evolution: phylogenetic inertia, adaptation and comparative methods. - J. Evol. Biol. 15: 899-910.

Burd, M. 1994. Bateman's principle and plant reproduction: the role of pollen limitation in fruit and seed set. - Bot. Rev. 60: 83-139.

Busch, J. W. 2005. The evolution of self-compatibility in geographically peripheral populations of Leavenworthia alabamica (Brassicaceae). - Am. J. Bot. 92: 503-1512.

Caccianiga, M., et al. 2006. The functional basis of a primary succession resolved by CSR classification. - Oikos 112: 10-20.

Charlesworth, D., et al. 1990. Inbreeding depression, genetic load, and the evolution of outcrossing rates in a multilocus system with no linkage. - Evolution 44: 1469-1489. Cheptou, P.-O., et al. 2000. Effects of competition on lifetime estimates of inbreeding depression in the outcrossing plant Crepis sancta (Asteraceae). - J. Evol. Biol. 13: 522-531. Cheptou, P.-O., et al. 2002. Mating system variation along a successional gradient in the allogamous and colonizing plant Crepis sancta (Asteraceae). - J. Evol. Biol. 15: 753-762. Cheptou, P.-O. and Massol, F. 2009. Pollination fluctuations drive evolutionary syndromes linking dispersal and mating system. - Am. Nat. 174: 46-55. 
Davis, H. G. 2005. r-selected traits in an invasive population. - Evol. Ecol. 19: 255-274.

Delmas, C., et al. 2014. High lifetime inbreeding depression counteracts the reproductive assurance benefit of selfing in a mass-flowering shrub. - BMC Evol. Biol. 14: 243.

Dornier, A., et al. 2008. Allee effect and self-fertilization in hermaphrodites: reproductive assurance in a structured metapopulation. - Evolution 62: 2558-2569.

Durka, W. and Michalski, S. G. 2012. Daphne: a dated phylogeny of a large European flora for phylogenetically informed ecological analyses. - Ecology 93: 2297-2297.

Eckert, C. G., et al. 2009. Plant mating systems in a changing world. - Trends Ecol. Evol. 25: $35-43$

Ellenberg, H., et al. 1991. Zeigerwerte von pflanzen in Mitteleuropa. - Ulmer.

Fisher, R. A. 1941. Average excess and average effect of a gene substitution. - Ann. Eugen. 11: $53-63$

Garamszegi, L. Z. 2014. Modern phylogenetic comparative methods and their application in evolutionary biology. - Springer.

Gelman, A. 2006. Prior distributions for variance parameters in hierarchical models (comment on article by Browne and Draper). - Bayesian Anal. 1: 515-534.

Grant, W. F. 1967. Cytogenetic factors associated with the evolution of weeds. - Taxon 16: 283-193.

Grime, J. P. 1974. Vegetation classification by reference to strategies. - Nature 250: 26-31.

Grime, J. P. 1977. Evidence for the existence of three primary strategies in plants and its relevance to ecological and evolutionary theory. - Am. Nat. 111: 1169-1194.

Grime, J. P. 1979. Plant strategies and vegetation processes. - Wiley.

Grime, J. P., et al. 2007. Comparative plant ecology - a functional approach to common British species (2nd edition). - Castlepoint Press.

Grime, J. P., et al. 1997. Integrated screening validates primary axes of specialisation in plants. - Oikos 79: 259-281.

Hadfield, J. 2014. MCMCgImm course notes. 
Hadfield, J. D. 2010. MCMC methods for multi-response generalized linear mixed models: the MCMCgImm R package. - J. Stat. Soft. 33: 1-22.

Hadfield, J. D. 2015. Increasing the efficiency of MCMC for hierarchical phylogenetic models of categorical traits using reduced mixed models. - Meth. Ecol. Evol. 6: 706-714.

Hadfield, J. D. and Nakagawa, S. 2010. General quantitative genetic methods for comparative biology: phylogenies, taxonomies and multi-trait models for continuous and categorical characters. - Journal Evol. Biol. 23: 494-508.

Hodgson, J. G., et al. 1999. Allocating C-S-R plant functional types: a soft approach to a hard problem. - Oikos 85: 282-294.

Huston, M. and Smith, T. 1987. Plant succession: life history and competition - Am. Nat. 130: 168-198.

Kalisz, S., et al. 2004. Context-dependent autonomous self-fertilization yields reproductive assurance and mixed mating. - Nature 430: 884-887.

Kattge, J., et al. 2011. TRY - a global database of plant traits. — Glob. Change Biol. 17: 29052935.

Klotz, S. 1984. Phytoökologische beiträge zur charakterisierung und gliederung urbaner ökosysteme, dargestellt am beispiel der Städte Halle und Halle-Neustadt. - Martin-LutherUniversität.

Knight, T. M., et al. 2005. Polle $-n$ limitation of plant reproduction: pattern and process. Annu. Rev. Ecol. Evol. Syst. 36: 467-497.

Kühn, I., et al. 2004. BiolFlor - a new plant trait database as a tool for plant invasion ecology. 10: $363-365$.

Lande, R. and Schemske, D. W. 1985. The evolution of self-fertilization and inbreeding depression in plants. I. Genetic models - Evolution 39: 24-40.

Lloyd, D. G. 1979. Some reproductive factors affecting the selection of self-fertilization in plants. - Am. Nat. 113: 67-79. 
Lloyd, D. G. 1992. Self-fertilization and cross-fertilization in plants. II. The selection of selffertilization. - Int. J. Plant. Sci. 153: 370-380.

McMullen, C. K. 1987. Breeding systems of selected Galapagos-islands angiosperms. - Am. J.

Bot. 74: 1694-1705.

Morgan, M. T., et al. 1997. The evolution of self-fertilization in perennials. - Am. Nat. 150: 618-638.

Navas, M. L., et al. 2010. Suites of plant traits in species from different stages of a Mediterranean secondary succession. - Plant. Biol. 12: 183-196.

Pannell, J. R. and Barrett, S. C. H. 1998. Baker's law revisited: reproductive assurance in a metapopulation. - Evolution 52: 657-668.

Price, S. C. and Jain, S. K. 1981. Are inbreeders better colonizers? - Oecologia 49: 283-286.

R Core Team. 2014. R: A language and environment for statistical computing. - R Foundation for Statistical Computing.

Raevel, V., et al. 2012. Mechanisms of ecological succession: insights from plant functional strategies. - Oikos 121: 1761-1770.

Raunkiaer, C. 1934. The life forms of plants and statistical plant geography. - Oxford University Press.

Renner, S. S. and Ricklefs, R. E. 1995. Dioecy and its correlates in the flowering plants. - Am. J. Bot. 82: 596-606.

Rydin, H. and Borgegard, S.-O. 1991. Plant characteristics over a century of primary sucession on islands: Lake Hjalmaren. — Ecology 72: 1089-1101

Sakai, A. K., et al. 1995. Biogeographical and ecological correlates of dioecy in the Hawaiian flora. - Ecology 76: 2530-2543.

Silvertown, J., et al. 2002. Ecological and genetic correlates of long-term population trends in the Park Grass experiment. - Am. Nat. 160: 409-420. 
Snell, R. and Aarssen, L. W. 2005. Life history traits in selfing versus outcrossing annuals:

exploring the 'time-limitation' hypothesis for the fitness benefit of self-pollination. - BMC

Ecol. 5.

Stebbins, G. L. 1950. Variation and evolution in plants. - Columbia University Press.

Stebbins, G. L. 1957. Self-fertilization and population variability in the higher plants. - Am.

Nat. 91: 337-354.

Stebbins, G. L. 1958. Longevity, habitat, and release of genetic variability in the higher plants.

- Cold Spring Harb. Symp. Quant. Biol. 23: 365-378.

Sutherland, S. 2004. What makes a weed a weed? Life history traits of native and exotic plants in the USA. - Oecologia 141: 24-39.

Uyenoyama, M., et al. 1993. Ecological and genetic factors directing the evolution of self-

fertilization. Oxford surveys in evolutionary biology. - Oxford University Press.

Van Kleunen, M. and Johnson, S. D. 2007. Effects of Self-Compatibility on the Distribution Range of Invasive European Plants in North America. - Conservation Biology 21(6): 15371544.

Violle, C., et al. 2007. Let the concept of trait be functional! - Oikos 116: 882-892.

Vogler, D. W. and Kalisz, S. 2001. Sex among the flowers: the distribution of plant mating systems. - Evolution 55: 202-204.

Westoby, M., et al. 2002. Plant ecological strategies: Some leading dimensions of variation between species. - Annu. Rev. Ecol. Syst. 33: 125-159. 
Table Legends

Table 1. Number of species per life form (columns), per CR level of the competitiveness index, and per S level of the stress-tolerance index (rows).

The levels of the competitiveness and stress-tolerance indexes are derived from CSR strategies as shown on Fig. 1.

Combinations of life form $(\mathrm{A}=$ annuals, $\mathrm{HP}=$ herbaceous perennials, $\mathrm{WP}=$ woody perennials $)$ and CSR strategy for each CR level are (i) A:R, A:SR, HP:R and $H P: S R$ in CR1, (ii) A:CR, A:CSR, A:S, HP:CR, HP:CSR, HP:S, WP:CSR and WP:S in CR2 and (iii) A:C, A:CS, HP:C, HP:CS, WP:C and WP:CS in CR3.

Combinations of life form and CSR strategy for each S level are (i) $A: C, A: C R, A: R, H P: C, H P: C R$, $H P: R$ and WP:C in S1, (ii) A:CSR, HP:CSR and WP:CSR in S2, (iii) A:CS, A:SR, HP:CS, HP:SR and WP:CS in S3, and (iv) A:S, HP:S and WP:S in S4. Combinations containing fewer than 10 species, which were excluded from GLMM analysis, are shown in italics.

\begin{tabular}{|c|c|c|c|c|c|}
\hline & & Annuals & $\begin{array}{l}\text { Herbaceous } \\
\text { perennials }\end{array}$ & $\begin{array}{c}\text { Woody } \\
\text { perennials }\end{array}$ & Total \\
\hline \multirow{3}{*}{ 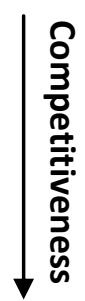 } & CR1 & 305 & 6 & 0 & 311 \\
\hline & CR2 & 251 & 648 & 15 & 914 \\
\hline & CR3 & 14 & 533 & 224 & 771 \\
\hline \multirow{4}{*}{ 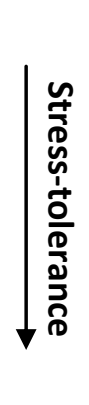 } & S1 & 413 & 332 & 180 & 925 \\
\hline & S2 & 34 & 472 & 11 & 517 \\
\hline & S3 & 110 & 279 & 44 & 433 \\
\hline & S4 & 13 & 104 & 4 & 121 \\
\hline
\end{tabular}

'This article is protected by copyright. All rights reserved.' 
Table 2. Relative proportions of mating systems at each level of the competitiveness index (CR levels 1 to 3), at each level of the stress-tolerance index (S levels 1 to 4), and for each life form. The proportions sum to 1 on each row, and the two highest proportions are shown in bold.

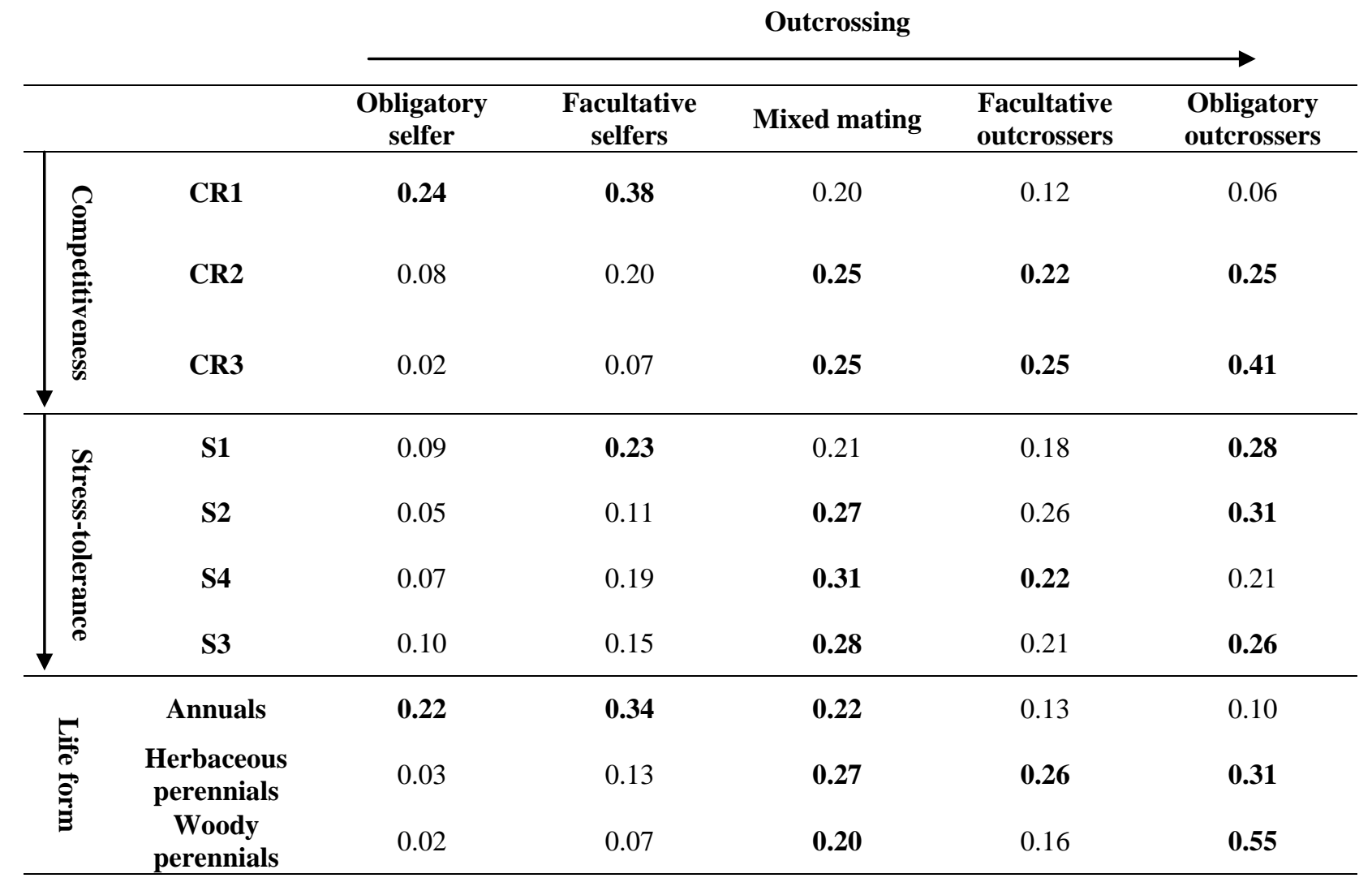


Table 3. Variation in mating system predicted by the GLMM model according to ecological strategies and life form. Outcrossing variation is shown for increasing competitiveness (a), stress tolerance (b), or depending on life form (c), from a row category to a column category. The $95 \%$ High Probable Density (HPD) interval of the GLMM model (in brackets) here represents the predicted outcrossing variation between these classes. A positive HPD interval means shift toward more outcrossing (denoted with " ${ }^{\prime \prime}$ ), while a negative HPD interval represents shift to more selfing (denoted with "\"). If a HPD interval includes 0 , there is no significant shift between the row and column classes (denoted with "=").

Significant shifts are shown in bold, except when a bound is very close to 0 (then in italics).

(a) Predicted outcrossing variation with increasing competitiveness

\begin{tabular}{|c|c|c|}
\hline From To & CR2 & CR3 \\
\hline CR1 & $\nearrow[1.71 ; 2.41]$ & $\nearrow[2.85 ; 3.88]$ \\
\hline CR2 & & $\nearrow[0.89 ; 1.65]$ \\
\hline
\end{tabular}

(b) Predicted outcrossing variation with increasing stress-tolerance

\begin{tabular}{|c|c|c|c|}
\hline From To & S2 & S3 & S4 \\
\hline S1 & $\nearrow[0.04 ; 0.73]$ & $=[-0.31 ; 0.38]$ & $=[-0.46 ; 0.58]$ \\
\hline S2 & & $\searrow[-0.69 ;-0.02]$ & $=[-0.77 ; 0.21]$ \\
\hline S3 & & & $=[-0.42 ; 0.60]$ \\
\hline
\end{tabular}

(c) Predicted outcrossing variation with life form

\begin{tabular}{|c|c|c|}
\hline From To & Herbaceous perennials & Woody perennials \\
\hline Annuals & $\nearrow[2.30 ; 3.00]$ & $\nearrow[2.65 ; 4.50]$ \\
\hline Herbaceous perennials & & $\nearrow[0.00 ; 1.74]$ \\
\hline
\end{tabular}




\section{Figure Legend}

Figure 1. Two basic indexes of competitiveness and of stress-tolerance are derived from the CSR triangle of ecological strategies (grey arrows). We projected the 7 CSR categories of the BiolFlor database onto the CR edge of the triangle to get the 3-level index of competitiveness, and projected the categories orthogonally to the CR edge to get the 4-level index of stress tolerance.

Along the index of increasing competitiveness, CR1 includes the R and SR strategies, CR2 includes CR, CSR and S strategies and CR3 includes C and SC strategies. Along the index of increasing stress-tolerance, S1 includes the R, CR and C strategies, S2 includes the CSR strategy, S3 includes the SR and SC strategies and S4 includes the S strategy.

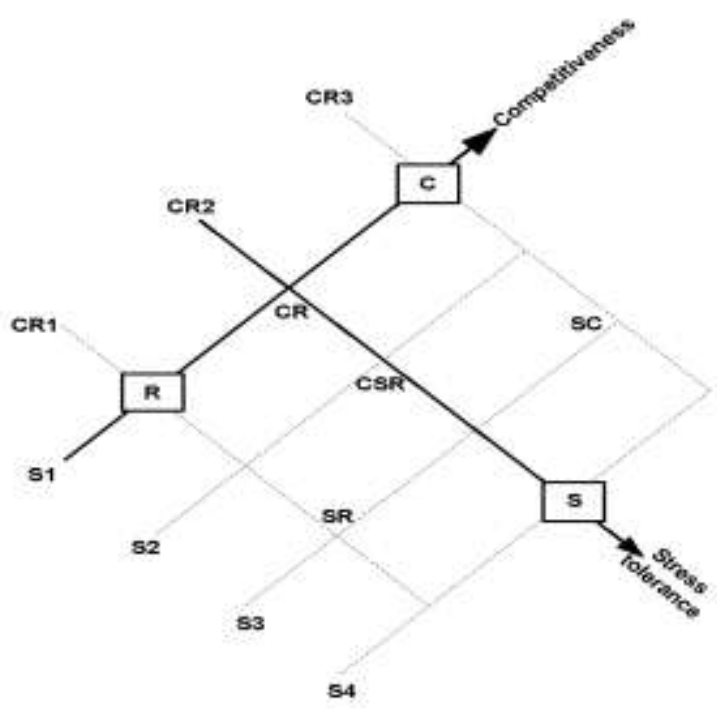

'This article is protected by copyright. All rights reserved.' 